\title{
The AJ072 antibody against the human transferrin receptor labels HeLa cells by surface immunofluorescence
}

Claudie Bian

Cell Physiology and Metabolism Dpt, University of Geneva, 1 rue Michel Servet, CH-1211, Geneva, Switzerland

\begin{abstract}
The AJ072 antibody against the human transferrin receptor labels the cell membrane of HeLa cells by surface immunofluorescence; AM236 does not.
\end{abstract}

\section{Introduction}

TfR (Transferrin receptor protein 1, CD71; Uniprot P02786) is a type II transmembrane glycoprotein that binds the iron-carrier glycoprotein transferrin (Tf). Cellular uptake of iron occurs via receptor-mediated endocytosis of diferric Tf/TfR complexes (Candelaria et al., 2021). Here, we describe the ability of the AJ072 recombinant antibody against human TfR1 to stain the cell membrane of HeLa cells by surface immunofluorescence; AM236 does not, presumably due to the fact that this antibody is poorly produced.

\section{Materials \& Methods}

\begin{abstract}
Antibodies: ABCD_AJ072 and ABCD_AM236 antibodies (https://web.expasy.org/abcd/, ABCD nomenclature) were produced by the Geneva Antibody Facility (https://www.unige.ch/medecine/antibodies/) as mini-antibodies with the antigen-binding $\mathrm{scFv}$ fused to a rabbit IgG Fc. The synthesized scFv sequences (GeneArt, Invitrogen) correspond to the sequences of the variable regions of the clones ch128.1 (for AJ072; Friden, 1994) and M16 (for AM236; Shusta and Tillotson, 2016) joined

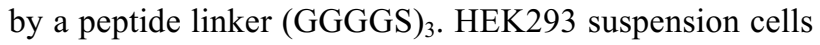
(growing in FreeStyle ${ }^{\text {TM }} 293$ Expression Medium, Gibco 12338) were transiently transfected with the vector coding for the scFv-Fc. Supernatants were collected after 4 days; both antibodies have low production yields $(<5 \mathrm{mg} / \mathrm{L})$.
\end{abstract}

Antigen: HeLa cells were cultured on glass coverslips (Menzel-Gläser, 22×22 mm) and grown in DMEM GlutaMAX $^{\mathrm{TM}}$ (Gibco 31966) supplemented with 8\% Fetal Bovine Serum (Gibco 10270).

Protocol: Cells were rinsed once with cold PBS, and kept for $30 \mathrm{~min}$ at $4{ }^{\circ} \mathrm{C}$ (ice+water bath). Cells were then incubated with the tested antibodies (undiluted, i.e. final concentration $5 \mathrm{mg} / \mathrm{L}$ in $\mathrm{PBS}+0.2 \%(\mathrm{w} / \mathrm{v}) \mathrm{BSA}(\mathrm{PBS}-$ $\mathrm{BSA})$ ) for $15 \mathrm{~min}$ at $4{ }^{\circ} \mathrm{C}$, fixed with $\mathrm{PBS}+4 \%$ paraformaldehyde (w/v) (Applichem A3013) for $15 \mathrm{~min}$ at room temperature, and blocked with $\mathrm{PBS}+40 \mathrm{mM}$ ammonium chloride $\left(\mathrm{NH}_{4} \mathrm{Cl}\right)$ (Applichem A3661) for 5 min. After 1 wash $(5 \mathrm{~min})$ with PBS-BSA, cells were incubated for $30 \mathrm{~min}$ with secondary goat anti-rabbit IgG conjugated to AlexaFluor-488 (1:300, Molecular Probes
A11034). After 3 washes (10 min) with PBS-BSA, cells were incubated during 5 min with DAPI (1:500, Molecular Probes D1306), washed twice with PBS-BSA and once with PBS, and mounted on slides (Menzel-Gläser, 76x26 $\mathrm{mm})$ with Möwiol (Hoechst) $+2.5 \%(\mathrm{w} / \mathrm{v})$ DABCO (Fluka 33480). Pictures were taken using a Zeiss LSM700 confocal microscope, with a $63 x$ Neofluar oil immersion objective.

\section{Results}

Using a cell surface labeling protocol, AJ072 successfully labeled the plasma membrane of HeLa cells (Fig. 1). No staining was seen with AM236 antibody; this might be due to the fact that this antibody is poorly produced. No staining was observed when the primary antibody was omitted (Fig. 1, No Ab).

\section{References}

Candelaria PV, Leoh LS, Penichet ML, Daniels-Wells TR. Antibodies targeting the Transferrin Receptor 1 (TfR1) as direct anti-cancer agents. Front Immunol. 2021; 12:607692. PMID: 33815364.

Friden, PM. Transferrin receptor reactive chimeric antibodies. USA; US6329508, 1994.

Shusta EV, Tillotson BJ. pH-dependent antibodies targeting the transferrin receptor and methods of use thereof to deliver a therapeutic agent. USA; US20170174778, 2016.

\section{Conflict of interest}

The authors declare no conflict of interest. 


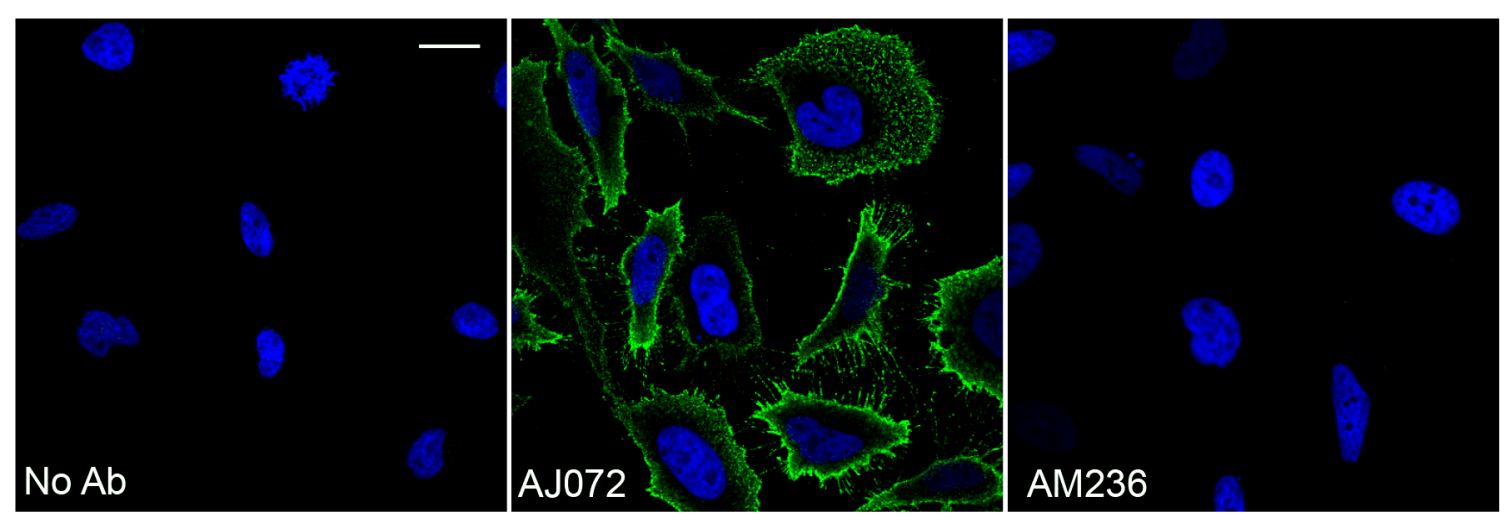

Fig. 1. AJ072 successfully labeled the cell membrane of HeLa cells (in green); in blue, nuclei were stained with DAPI. No labelling was seen for AM236 antibody, or when the primary antibody was omitted (No Ab panel). Scale bar: $20 \mu \mathrm{m}$. 